\title{
Effect of stretching exercises versus autogenic training on preeclampsia
}

\author{
Mohamed A. Awad', Marwa E. Hasanin', Mona Mohamed Taha ${ }^{2,3, *}$, Amir A. Gabr ${ }^{4}$ \\ 'Department of Physical Therapy for Women's Health, Faculty of Physical Therapy, Cairo University, Cairo, Egypt \\ ${ }^{2}$ Department of Physical Therapy for Cardiopulmonary Disorders and Geriatrics, Faculty of Physical Therapy, Cairo University, Cairo, Egypt \\ ${ }^{3}$ Department of Rehabilitation, College of Health and Rehabilitation Sciences, Princess Nourah bint Abdulrahman University, Riyadh, Saudi Arabia \\ ${ }^{4}$ Department of Obstetrics and Gynecology, Faculty of Medicine, Cairo University, Cairo, Egypt
}

Preeclampsia (PE) is the most common medical complication of pregnancy characterized by hypertension and significant proteinuria after the 20th week of gestation, its prevalence is about $2 \%-8 \%$ of pregnancies. Antihypertensive drugs were found to have an adverse effect to both the mother and the fetus so interest is increased in nonchemical treatment. This study was conducted to compare between the effects of stretching exercises versus autogenic training (AT) on PE. This study was carried out on 40 preeclamptic primiparous women, their gestational age was exceeding 20 weeks. They were randomly divided into two equal groups; group A consisted of 20 women received stretching exercises and group $B$ consisted of 20 women received relaxation training in the form of AT. All patients in both groups A and B received (3 sessions per week for 6 weeks) and received methyldopa as the antihy- pertensive drug. Evaluation of all patients in both groups $A$ and $B$ was done before and after the treatment program by assessing arterial blood pressure and proteinuria. Results of this study revealed that there is a significant decrease in systolic blood pressure, diastolic blood pressure and proteinuria in both groups $A$ and $B$ after 6 weeks of treatment. There was no significant difference between both groups posttreatment in systolic blood pressure, diastolic blood pressure, and proteinuria. It can be concluded that both stretching exercise and AT were found to be effective nonchemical methods which control the symptoms of PE.

Keywords: Stretching exercise, Autogenic training, Pre-eclampsia, Blood pressure, Proteinuria

\section{INTRODUCTION}

The actual cause of preeclampsia (PE) is unknown, however, some evidence suggests that PE occurs due to vascular endothelial cell dysfunction resulting in decreasing the synthesis of vasodilators like prostacyclin and nitric oxide which will lead to vasospasm resulting in hypertension (Witlin et al., 2000). PE is characterized by sudden onset of raise in the blood pressure and protein in urine in the second half of pregnancy, resulting in $22 \%$ of maternal deaths and $18 \%$ of all premature babies, and increases the risk that the mother may have cardiovascular disease in the future (Anderson, 2007).

Stretching exercise was found to regulate the action of the autonomic nervous system. The use of stretching exercise during preg- nancy can stimulate mechanoreceptors of the skeletal muscles which regulate parasympathetic and sympathetic activities because stretching the muscles to their fullest length will release stress (Motivala et al., 2006). In a study done to compare the effect of stretching or walking on the incidence of PE, it was found that women in the daily stretching program experienced a significantly lower incidence of PE (Yeo et al., 2008).

Stress factors are involved to great extent in hypertension, so psychological techniques as relaxation have been used to control the elevated blood pressure. Many hypertensive patients tried alternatives and many studies supported the use of biofeedback, autogenic training (AT), and yoga (Ernst, 2005). AT is one of the nonpharmacologic treatments that are very effective in lowering blood pressure (Watanabe et al., 2003), as it can decrease the ele-
*Corresponding author: Mona Mohamed Taha

(iD) https://orcid.org/0000-0001-9080-5061

Department of Physical Therapy for Cardiopulmonary Disorders and Geriatrics, Faculty of Physical Therapy, Cairo University, 2-street 9, Helwan, Cairo 11722, Egypt E-mail: Monady2007@yahoo.com

Received: October 19, 2018 /Accepted: December 22, 2018
This is an Open Access article distributed under the terms of the Creative Commons Attribution Non-Commercial License (http://creativecommons.org/licenses/by-nc/4.0/) which permits unrestricted non-commercial use, distribution, and reproduction in any medium, provided the original work is properly cited. 
vated blood pressure by an average of $10 \mathrm{mmHg}$ in the systole and $5 \mathrm{mmHg}$ in the diastole (Herrmann, 2002).

Unfortunately, the literature review was unable to identify any study comparing the effect of stretching exercise and AT as nonpharmacological treatment methods in alleviating the PE symptoms. Consequently, the purpose of this study was to compare the effect of those two methods for treating PE.

\section{MATERIALS AND METHODS}

A randomized, parallel group study was conducted at the outpatient clinics of Cairo University Hospital, Egypt. Ethical approval was obtained from the Institutional Review Board at Faculty of physical therapy, Cairo University before study commencement No: P.T REC/012/002068. The women participated in the study after signing an informed consent form before data collection. Forty mild preeclamptic primiparous women participated in this study, their ages were ranged from 25-35 years old, their gestational age was exceeding 24 weeks' gestation and their body mass index did not exceed $35 \mathrm{~kg} / \mathrm{m}^{2}$. Women with cardiorespiratory diseases, diabetes mellitus, twins, previous history of preterm labor or repeated abortion as well as bleeding and intra uterine fetal growth anomalies or retardation were excluded from the study. The women were randomly assigned to group $A(n=20)$ or group $B(n=20)$ by an independent person blinded to the research protocol and not involved in the trial who selected numbers from sealed envelopes containing numbers chosen by a random number generator. The randomization was restricted to permuted blocks to ensure that equal numbers were allocated to each group.

\section{Evaluative procedures}

\section{Measurement of arterial blood pressure}

In both groups $\mathrm{A}$ and $\mathrm{B}$, mercury sphygmomanometer and stethoscope were used to measure arterial blood pressure from the right arm while the preeclamptic woman was in half lying position, three trials for measurement of the blood pressure was taken then the mean was calculated before and after the end of the treatment (6 weeks).

\section{Measurement of proteinuria}

Each preeclamptic woman in both groups A and B was asked to collect a 24-hr clean midstream urine sample after cleaning the vulva with current water in sterilized glass bottles for measurement of protein level in urine before and after the end of the treatment (6 weeks).

\section{Treatment procedures}

The patients in both groups completed the treatment program for 6 weeks ( 3 sessions per week). Group A received stretching exercises and group $B$ received relaxation training in the form of AT. The antihypertensive drug (Methyldopa) was given for both groups $\mathrm{A}$ and $\mathrm{B}$ all through the study period, the dose of the drug varies from one patient to another according to her state to keep the blood pressure at a safer level for each case.

\section{Stretching exercise}

Warm-up and cool down were done in the form of costal breathing exercise. Stretching exercise was done for pectoralis major, neck, back, hip adductor, hamstring, calf and hip flexors muscles. Each patient performed the stretching exercises under the supervision of the physical therapist. Stretching exercise for selected muscles: was done as follow: 10-sec stretching of each muscle followed by 10 -sec relaxation in the 1st 2 weeks, then 15 -sec stretching of each muscle followed by 15 -sec relaxation in the next 2 weeks of treatment (3rd and 4th weeks) and in the last 2 weeks of treatment (5th and 6th weeks), 20-sec stretching of each muscle followed by 20-sec relaxation. Repetition of each stretching exercise was 6 times. After the end of the program of treatment (6 weeks), each woman was instructed to do the same exercises of stretching in the remaining duration of pregnancy until delivery.

\section{Relaxation procedures (Autogenic training)}

Relaxation procedures were performed in the following steps: first step - preparation: Each preeclamptic woman was instructed about this method of treatment and its importance for reducing blood pressure to gain her confidence and cooperation during treatment. In a quiet room with light dim, each preeclamptic woman was advised to evacuate her bladder and then to assume the half lying position with using light pillows to fill all her body curvatures and her arms rested on the plinth to be completely relaxed with no restrictive clothes. Second step - AT: The session began with a few minutes of mental relaxation as the patient was asked to imagine herself in a lovely place that makes her relaxed. Each session included 18 exercises combining both relaxation and auto-suggestion (limb heaviness exercise, limb warmth exercise, cardiac exercise, respiration exercise, solar plexus warmth exercise, and "forehead cooling" exercise). Each exercise was in the form of a group of phrases. Each phrase was recited by the physical therapist in a slow calm and soothing tone and then repeated, mentally or vocally, by the learner, About $30 \mathrm{sec}$ was allowed for each exercise and a further $30-40 \mathrm{sec}$ was allowed for continuing attention 
focusing by the patient. Third step - termination: After finishing the 18 exercises of the AT each preeclamptic woman was asked to make a few fists with her hands (10 times), bend and stretch her elbows for (10 times), then her knees for (10 times), gently stretch her body on the plinth, roll on the plinth to be inside lying position and slowly get up until sitting position, this allows her to make a gradual return to her normal activity.

\section{Sample size}

Based on the pilot study, the sample size was calculated according to the difference in the mean value of systolic blood pressure between group A (148.5 \pm 8.8$)$ and group B $(156.5 \pm 6.6)$ measured pretreatment, with an effect size of 1.02 . Assuming $\alpha=0.05$, power of $80 \%$, so a sample size of 16 patients per group would be required (G Power 301; http://www.psycho.uni-duesseldorf.de). We recruited 40 subjects to account for the dropout rates.

\section{Statistical analysis}

Results are expressed as mean \pm standard deviation. Test of normality, Kolmogorov-Smirnov test, was used to measure the distribution of data measured pre-treatment. Accordingly, the comparison between variables in the two groups was performed using unpaired $t$-test. Comparison between variables measured before and after treatment in the same group was performed using paired $t$-test. IBM SPSS Statistics ver. 19.0 (IBM Co., Armonk, NY, USA) was used for data analysis. $P$-value of $\leq 0.05$ was considered significant.

\section{RESULTS}

\section{Systolic blood pressure}

There was a statistically significant decrease in systolic blood pressure in both groups A and B posttreatment. When comparing both groups together, there was no statistically significant difference between both groups' pretreatment and posttreatment (Table 1).

\section{Diastolic blood pressure}

There was a statistically significant decrease in diastolic blood pressure in both groups A and B posttreatment. When comparing both groups together, there was no statistically significant difference between both groups' pretreatment and posttreatment (Table 2).

\section{Proteinuria}

There was a statistically significant decrease in proteinuria in both groups A and B posttreatment. When comparing both
Table 1. Inter- and intragroup comparison between mean values of systolic blood pressure $(\mathrm{mmHg})$ in the two studied groups measured before and after treatment

\begin{tabular}{lcccc}
\hline Variable & Group $A(\mathrm{n}=20)$ & Group $B(\mathrm{n}=20)$ & $t$-value & $P$-value \\
\hline Pretreatment & $153.00 \pm 5.48$ & $150.25 \pm 6.78$ & 1.411 & 0.166 \\
Posttreatment & $132.00 \pm 5.48$ & $130.75 \pm 3.35$ & 0.870 & 0.391 \\
$\%$ Change & $13.73 \downarrow \downarrow$ & $12.98 \downarrow \downarrow$ & & \\
$t$-value & 13.424 & 10.773 & & \\
$P$-value & 0.001 & 0.01 & & \\
\hline
\end{tabular}

Values are presented as mean \pm standard deviation.

Group A, 20 women received stretching exercises; Group B, 20 women received relaxation training.

Table 2. Inter- and intragroup comparison between mean values of diastolic blood pressure $(\mathrm{mmHg})$ in the two studied groups measured before and after treatment

\begin{tabular}{lcccc}
\hline Variable & Group $A(\mathrm{n}=20)$ & Group $B(\mathrm{n}=20)$ & $t$-value & $P$-value \\
\hline Pretreatment & $99.00 \pm 7.00$ & $102.25 \pm 5.95$ & -1.582 & 0.122 \\
Posttreatment & $86.25 \pm 7.05$ & $88.75 \pm 4.55$ & -1.333 & 0.192 \\
$\%$ Change & $12.88 \downarrow \downarrow$ & $13.20 \downarrow \downarrow$ & & \\
$t$-value & 15.022 & 11.171 & & \\
$P$-value & 0.001 & 0.01 & & \\
\hline
\end{tabular}

Values are presented as mean \pm standard deviation.

Group A, 20 women received stretching exercises; Group B, 20 women received relaxation training

Table 3. Inter- and intragroup comparison between mean value of proteinuria $(\mathrm{g} / 24 \mathrm{hr})$ in the two studied groups measured before and after treatment

\begin{tabular}{lcccc}
\hline Variable & Group $\mathrm{A}(\mathrm{n}=20)$ & Group $\mathrm{B}(\mathrm{n}=20)$ & $t$-value & $P$-value \\
\hline Pretreatment & $2.41 \pm 1.02$ & $2.33 \pm 1.01$ & 0.262 & 0.795 \\
Posttreatment & $1.94 \pm 0.78$ & $1.93 \pm 0.91$ & 0.056 & 0.956 \\
$\%$ Change & $19.50 \downarrow \downarrow$ & $17.17 \downarrow \downarrow$ & & \\
$t$-value & 4.229 & 3.899 & & \\
$P$-value & 0.001 & 0.001 & & \\
\hline
\end{tabular}

Values are presented as mean \pm standard deviation.

Group A, 20 women received stretching exercises; Group B, 20 women received relaxation training.

groups together, there was no statistically significant difference between both groups' pretreatment and posttreatment (Table 3).

\section{DISCUSSION}

Results of this study showed that there is a significant decrease in systolic blood pressure, diastolic blood pressure and proteinuria in both groups A and B after 6 weeks of treatment. There was no significant difference between both groups posttreatment in systolic blood pressure, diastolic blood pressure, and proteinuria. It was found that exercises like prenatal stretching exercises (PSEs), 
tai chi or yoga may lead to a great effect on autonomic nervous system responses as reducing stress but few studies concentrated on examining the effects of PSEs (Jallo et al., 2008; Lu and Kuo, 2003).

The results of the current study came in accordance with (Bahadoran et al., 2015) who demonstrated that the effect of stretching exercise superimposed the effect of walking in reducing systolic and diastolic blood pressure in the second and third trimester of pregnancy. The findings of the current study are consistent with (Yeo, 2009) who showed that women practiced PSE as a daily routine experienced a significantly lower incidence of developing PE.

Physical activity increases parasympathetic activity and that lead to bradycardia, exercise could considerably decrease preeclampsia risk by lowering blood pressure and resting heart rate (Carter et al., 2003). Moderate to vigorous leisure-time physical activities before and during pregnancy led to a $35 \%$ reduction in the occurrence of PE (Saftlas et al., 2004). Exercise also may protect against PE by reducing the concentration of oxidative substances which is produced by the pregnant woman due to stress. It also stimulates placental vascularity and growth and prevents its endothelial dysfunction (Falcao et al., 2010).

Muscle sympathetic nerve activity was measured at 22nd and 33 rd weeks of pregnancy and at the postnatal period and it was found to be significantly increased. Some pregnant women may induce sympathetic over activity and may develop PE. Based on those results, it was suggested that PE develops when there is failure in the normal vasodilatation mechanism. PSE may inhibit or decrease this failure through sending signals from stretched skeletal muscles to the central nervous system improving the vagal tone (Fischer et al., 2004) or create a state of vagal preponderance (parasympathetic over activity) (Martens et al., 2008).

Stretching also lead to replacement of the necrotic tissue by fibroblasts and collagen and opposes the pathologic concentric hypertrophy which occurs with chronic hypertension (Wakatsuki et al., 2004). Regarding the effect of AT on PE symptoms, these results match those observed in earlier study by (Schwickert et al., 2006) who reported that relaxation techniques were very effective in reducing hypertension (systolic and diastolic blood pressures), heart rate and respiratory rate (Schwickert et al., 2006). The effect of relaxation in reducing blood pressure could be linked to its effect in lowering in the sympathetic response of the hypothalamus which was expected to decrease the blood pressure. The release of tension in the skeletal muscles gained by relaxation raises the peripheral blood flow which will lead to the lowering of the blood pressure and heart rate and lead to slower deeper breathing. Also, relaxation response gets over the effects of sympathetic activity by enhancing the action of the parasympathetic nervous system. When there is a lower in the sympathetic activity, there will be a reduction in plasma renin-angiotensin activity and aldosterone concentration leading to lowering in the blood pressure (Broms, 1999).

In a general, Halligan et al. (1997) explained that there was a direct relationship between the blood pressure and proteinuria in $\mathrm{PE}$ and the obvious decline of proteinuria in both groups could be attributed to the very highly significant decrease that occurred in systolic blood pressure and diastolic blood pressures in these groups. Further studies are needed to compare the effect of different nonpharmacological treatment methods for PE treatment and prevention.

Our study findings showed that both stretching exercise and AT were effective nonchemical procedures in alleviating the symptoms of PE through decreasing the elevated blood pressure and proteinuria.

\section{CONFLICT OF INTEREST}

No potential conflict of interest relevant to this article was reported.

\section{ACKNOWLEDGMENTS}

We thank the women who participated in this study.

\section{REFERENCES}

Anderson CM. Preeclampsia: exposing future cardiovascular risk in mothers and their children. J Obstet Gynecol Neonatal Nurs 2007;36:3-8.

Bahadoran P, Pouya F, Zolaktaf V, Taebi M. The effect of stretching exercise and walking on changes of blood pressure in nulliparous women. Iran J Nurs Midwifery Res 2015;20:205-210.

Broms C. Free from stress by autogenic therapy. Relaxation technique yielding peace of mind and self-insight. Lakartidningen 1999;96:588592.

Carter JB, Banister EW, Blaber AP. Effect of endurance exercise on autonomic control of heart rate. Sports Med 2003;33:33-46.

Ernst E. Complementary/alternative medicine for hypertension: a minireview. Wien Med Wochenschr 2005;155:386-391.

Falcao S, Bisotto S, Michel C, Lacasse AA, Vaillancourt C, Gutkowska J, Lavoie JL. Exercise training can attenuate preeclampsia-like features 
in an animal model. J Hypertens 2010;28:2446-2453.

Fischer T, Schobel HP, Frank H, Andreae M, Schneider KT, Heusser K. Pregnancy-induced sympathetic overactivity: a precursor of preeclampsia. Eur J Clin Invest 2004;34:443-448.

Halligan AW, Shennan A, Lambert PC, Bell SC, Taylor DJ, de Swiet M. Automated blood pressure measurement as a predictor of proteinuric pre-eclampsia. Br J Obstet Gynaecol 1997;104:559-562.

Herrmann JM. Essential hypertension and stress. When do yoga, psychotherapy and autogenic training help? MMW Fortschr Med 2002;144: 38-41.

Jallo N, Bourguignon C, Taylor AG, Utz SW. Stress management during pregnancy: designing and evaluating a mind-body intervention. Fam Community Health 2008;31:190-203.

Lu WA, Kuo CD. The effect of Tai Chi Chuan on the autonomic nervous modulation in older persons. Med Sci Sports Exerc 2003;35:1972-1976.

Martens A, Greenberg J, Allen JJ. Self-esteem and autonomic physiology: parallels between self-esteem and cardiac vagal tone as buffers of threat. Pers Soc Psychol Rev 2008;12:370-389.

Motivala SJ, Sollers J, Thayer J, Irwin MR. Tai Chi Chih acutely decreases sympathetic nervous system activity in older adults. J Gerontol A Biol Sci Med Sci 2006;61:1177-1180.

Saftlas AF, Logsden-Sackett N, Wang W, Woolson R, Bracken MB. Work, leisure-time physical activity, and risk of preeclampsia and gestational hypertension. Am J Epidemiol 2004;160:758-765.

Schwickert M, Langhorst J, Paul A, Michalsen A, Dobos GJ. Stress management in the treatment of essential arterial hypertension. MMW Fortschr Med 2006;148:40-42.

Wakatsuki T, Schlessinger J, Elson EL. The biochemical response of the heart to hypertension and exercise. Trends Biochem Sci 2004;29:609617.

Watanabe Y, Cornélissen G, Watanabe M, Watanabe F, Otsuka K, Ohkawa S, Kikuchi T, Halberg F. Effects of autogenic training and antihypertensive agents on circadian and circaseptan variation of blood pressure. Clin Exp Hypertens 2003;25:405-412.

Witlin AG, Saade GR, Mattar F, Sibai BM. Predictors of neonatal outcome in women with severe preeclampsia or eclampsia between 24 and 33 weeks' gestation. Am J Obstet Gynecol 2000;182:607-611.

Yeo S. Adherence to walking or stretching, and risk of preeclampsia in sedentary pregnant women. Res Nurs Health 2009;32:379-390.

Yeo S, Davidge S, Ronis DL, Antonakos CL, Hayashi R, O'Leary S. A comparison of walking versus stretching exercises to reduce the incidence of preeclampsia: a randomized clinical trial. Hypertens Pregnancy 2008;27:113-130. 